\title{
A Method for Calculating the Configuration Factor between a Flame and a Receiving Target for a Wide Range of Flame Geometries Relevant to Large Scale Fires
}

\section{G. HANKINSON}

British Gas Corporation Research and Development Division

Midlands Research Station

Wharf Lane, Solihull

West Midlands, England B91 2JW

\section{ABSTRACT}

To predict the thermal radiation field surrounding a fire, it is necessary to know the configuration factor between the flame and the recelving target. This requirement has previously imposed restrictions on the range of geometries that could be selected to represent the shape of flames. A method has therefore been developed which will enable the configuration factor to be calculated for any flame shape and has been applied to a particular geometrical conflguration that can be used to represent a wide range of flame shapes associated with large fires. The method also allows the alignment of a target that is subjected to the maximum incident thermal radiation to be identified. This is of particular interest when studying radiant heat transfer.

\section{INTRODUCTION}

Radiative heat transfer from the flames associated with large fires can endanger objects located near the flame. A quantitative determination of the thermal radiation field surrounding a fire is, therefore, necessary and this involves four major steps:

1. Specification of the size and shape of the flame.

2. Specification of the thermal radiation properties of the flame. In many cases flames are considered to emit uniformly over the whole of their surface.

3. Determination of the transmissivity of the intervening atmosphere. This will depend upon the atmospheric conditions, the emission characteristics of the flame surface and the mean path length from the object to the flame surface.

4. Calculation of the configuration factor. This enables the relative position and geometry of the flame and the receiving object to be taken into account.

The shapes of flames associated with large hydrocarbon pool fires have been studied by several groups of workers. These include American Gas Association for spills into $1.83,6.10$ and $24.38 \mathrm{~m}$ diameter earthen bunds, Raj et a1. 2, U.S. Department of Transportation, for spills onto water, Mizner and Eyre ${ }^{3}$, Shell, for spills into $20 \mathrm{~m}$ dlameter bunds and Moorhouse ${ }^{4}$, British Gas Corporation for spills into rectangular bunds ranging in area from 37.2 to $185.8 \mathrm{~m}^{2}$ and length to width ratio from 1 to 2.5 . The flames assoctated with 
these large pool fires were approximated using regular geometrical shapes. However, restrictions on the geometries adopted were imposed, in some cases, by the techniques available for calculating configuration factors between the flame and a target near the flame. The flame shapes defined in these studies range from an oblique cylinder of circular cross section, a tilted cylinder of circular cross section and an oblique cylinder of elliptical cross section.

Analytical expressions for calculating configuration factors have been derived using the geometrical determination technique ${ }^{5}$ or the contour integral method $^{6}$. Such equations are available for oblique cylinders of circular cross section and other simple shapes but are restricted to certain locations and orientations of the target. The contour integral method can also be used as a numerical technique, however, the identification of the contour that encloses that part of the flame surface contained within the field-of-view of the target can be extremely complex.

Another technique developed by Rein et $a 1^{7}$ for tilted cylinders and recelving targets located at ground level directly downind of the flame is an area integral method. That part of the surface of the cylinder contained within the field-of-view of the target is divided into small parallelograms. Unfortunately the method makes no allowances for the differences in area of these small parallelograms as their position changes around the circumference of the cylinder. The expression used to calculate the area is accurate only for elements located normal to the wind direction on the upwind or downwind edges of the flame, or for the situation in which the angle of tilt of the flame from the vertical is zero. Inaccuracies using this method increase as the flame tilt increases and will further increase if the method is extended so that calculations can be performed for targets located at positions crosswind of the fire.

An area integral method has, therefore, been developed which overcomes the above difficulties and also extends the range of application to cover any solid geometrical shape. This is accomplished by dividing the whole of the surface of the solid shape used to represent the flame into small triangular elements. Conditions are then employed to select only those elements that can be seen from the position and orientation of the target. In some applications it is of great benefit to know the alignment of the target at a particular location that is subjeted to the maximum incident thermal radiation. When the emissive power of the flame is assumed to be uniform over the surface, this is identical to the alignment of the target that subtends the maximum configuration factor. A technique which enables this orientation of the target to be determined has been included in the method.

\section{INCIDENT THERMAL RADIATION}

The thermal radiation incident upon a target, 1 , at any position and orlentation relative to an emitting surface, 2 , is dependent upon the surface emissive power, the transmissivity of the atmosphere between the surface and the target and the configuration factor as follows:

$I=\mathrm{E} \mathrm{F}_{21} \mathrm{C}$

where I represents the incident thermal radiation $\left(\mathrm{kWm}^{-2}\right)$

$\mathrm{E}$ represents the surface emissive power $\left(\mathrm{kWm}^{-2}\right)$

$F_{21}$ represents the configuration factor

$\tau^{21}$ represents the transmissivity of the atmosphere. 


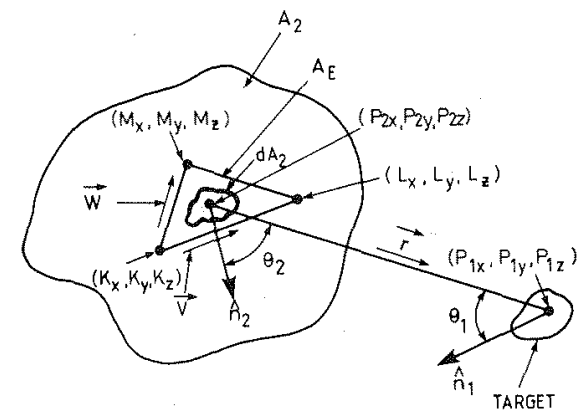

FIGURE 1. Radiant heat transfer between a finite surface and an infinitesimal target.

\section{CONFIGURATION FACTOR}

The configuration factor for radiant heat transfer between an emitting surface, 2, of any shape and an infinitesimal target, 1 , is given, with reference to $\mathrm{Fig} \cdot 1$, as follows:

$F_{21}=\int \frac{\cos \theta_{1} \cos \theta_{2} \mathrm{dA}_{2}}{\pi \mathrm{A}^{2}}$

where $A_{2}$ represents the surface area of that part of the emitting surface that is both contained within the field-of-view of the target and contains the target within its own field-of-view.

To enable Eq. (2) to be solved by a numerical integration technique it is necessary to define the geometry of the surface. This, in general, can be accomplished by dividing the whole of the surface area into small triangular elements and specifying the position in space of the nodal points relative to a set of Cartesian axes.

The configuration factor for radiant heat transfer between a small triangular element, $E$, and an infinitesimal target is given, with reference to Fig. 1, as follows:

$\mathrm{F}_{\mathrm{El}}=\frac{\cos \theta_{1} \cos \theta_{2} \mathrm{~A}_{\mathrm{E}}}{\pi \mathrm{r}^{2}}$

where $A_{E}$ represents the area of the triangular element.

$A_{E}$ is obtained from the vector product of the two vectors $\vec{V}$ and $\vec{W}$ which form two sides of the triangular element. $\vec{V}$ and $\vec{W}$ are obtained from the positions of the nodal points as follows:

$\vec{V}=\left(V_{x}, V_{y}, V_{z}\right)=\left[\left(L_{x}-K_{x}\right),\left(L_{y}-K_{y}\right),\left(L_{z}-K_{z}\right)\right]$
$\vec{W}=\left(W_{x}, W_{y}, W_{z}\right)=\left[\left(M_{x}-K_{x}\right),\left(M_{y}-K_{y}\right),\left(M_{z}-K_{z}\right)\right]$ 
This gives:

$A_{E}=\frac{|\vec{V} x \vec{W}|}{2}=\frac{\left[\left(V_{y} W_{z}-V_{z} W_{y}\right)^{2}+\left(V_{z} W_{x}-v_{x} W_{z}\right)^{2}+\left(v_{x} W_{y}-V_{y} W_{x}\right)^{2}\right]^{\frac{1}{2}}}{2}$

The length $r$ of the connecting line between the centre-of-area of the small triangular element, $E$, and the target, 1 , is the magnitude of the vector $\vec{r}$ which can be expressed in terms of the positions of the end points as follows:

$\vec{r}=\left(r_{x}, r_{y}, r_{z}\right)=\left[\left(P_{1 x}-P_{2 x}\right),\left(P_{1 y}-P_{2 y}\right),\left(P_{1 z}-P_{2 z}\right)\right]$

where $\quad\left(P_{2 x}, P_{2 y}, P_{2 z}\right)$ represents the centre-of-area of the

$\left(P_{2 x}, P_{2 y}, P_{2 z}\right)=\left[\frac{\left(K_{x}+L_{x}+M_{x}\right)}{3}, \frac{\left(K_{y}+L_{y}+M_{y}\right)}{3}, \frac{\left(K_{z}+L_{z}+M_{z}\right)}{3}\right]$

and $\quad\left(P_{1 x}, P_{1 y}, P_{l_{z}}\right)$ specifies the position of the target.

Therefore $r$ is given by:

$r=|\vec{r}|=\left[r_{x}^{2}+r_{y}^{2}+r_{z}^{2}\right]^{\frac{1}{2}}$

To obtain the values of $\cos \theta_{1}$ and $\cos \theta_{2}$ it is necessary to define unit vectors, $\hat{r}_{12}$ and $\hat{r}_{21}$, that 11 e along the connecting line and are directed respectively from the triangular element to the target and vice versa. It is also necessary to define the unit normal, $\hat{n}_{1}$, to the target and to calculate the outward unit normal, $\hat{n}_{2}$ to the triangular element.

$\hat{r}_{12}=\left[\frac{\left(P_{1 x}-P_{2 x}\right)}{r}, \frac{\left(P_{1 y}-P_{2 y}\right)}{r}, \frac{\left(P_{1 z}-P_{2 z}\right)}{r}\right]$

$\hat{r}_{21}=\left[\frac{\left(P_{2 x}-P_{1 x}\right)}{r}, \frac{\left(P_{2 y}-P_{1 y}\right)}{r}, \frac{\left(P_{2 z}-P_{1 z}\right)}{r}\right]$

$\hat{\mathrm{n}}_{2}$ is obtained in a similar manner to $\mathrm{AE}_{\mathrm{E}}$ from the vector product of the two vectors $\vec{V}$ and $\vec{W}$ as follows:

$\hat{\mathrm{n}}_{2}=\frac{\overrightarrow{\mathrm{V}} \times \overrightarrow{\mathrm{W}}}{|\overrightarrow{\mathrm{V}} \times \overrightarrow{\mathrm{W}}|}=\left[\frac{\left(V_{y} W_{z}-V_{z} W_{y}\right)}{2 A_{E}}, \frac{\left(V_{z} W_{x}-V_{x} W_{z}\right)}{2 A_{E}}, \frac{\left(V_{x} W_{y}-V_{y} W_{x}\right)}{2 A_{E}}\right]$

$\hat{\mathrm{n}}_{\mathrm{I}}$ specifies the orientation of the target and can be written as:

$\hat{\mathrm{n}}_{1}=\left(\mathrm{n}_{1 \mathrm{x}}, \mathrm{n}_{1 \mathrm{y}}, \mathrm{n}_{1 z}\right)$

$\cos \theta_{1}$ and $\cos \theta_{2}$ are determined from the scaler product of the unit vectors $\hat{\mathrm{r}}_{21}$ and $\hat{\mathrm{n}}_{1}$, and $\hat{\mathrm{r}}_{12}$ and $\hat{\mathrm{n}}_{2}$ respectively.

$\cos \theta_{1}=\hat{n}_{1} \cdot \hat{r}_{21}=\left[\frac{n_{1 x}\left(P_{2 x}-P_{1 x}\right)}{r}+\frac{n_{1 y}\left(P_{2 y}-P_{1 y}\right)}{r}+\frac{n_{1 z}\left(P_{2 z}-P_{1 z}\right)}{r}\right]$ 


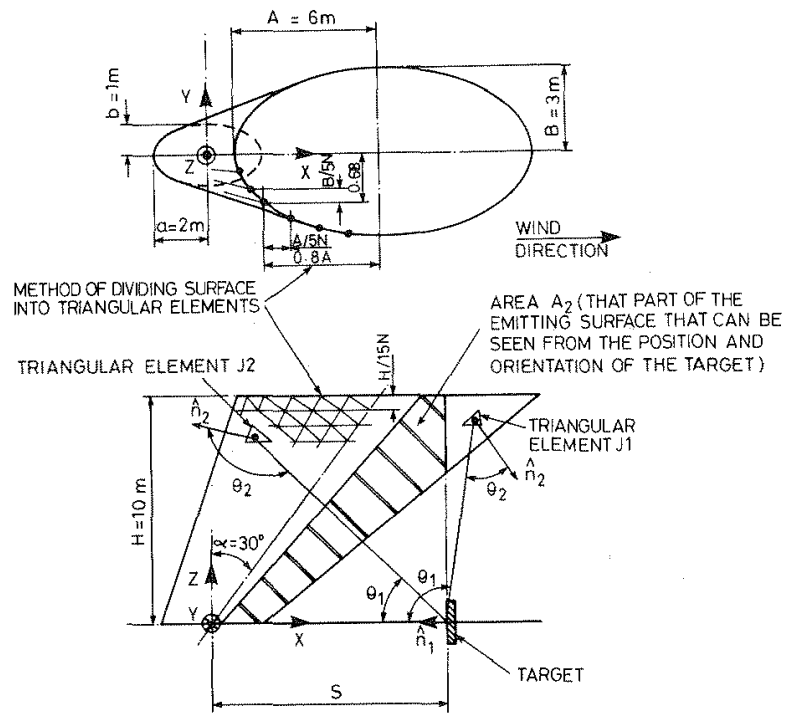

FIGURE 2. An oblique conical frustum of elliptical cross section showing the method used to define the area $\mathrm{A}_{2}$.

$$
\begin{aligned}
\cos \theta_{2}=\hat{n}_{2} \cdot \hat{r}_{12}= & {\left[\frac{\left(V_{y} W_{z}-V_{z} W_{y}\right)\left(P_{1 x}-P_{2 x}\right)}{2 A_{E} r}+\frac{\left(V_{z} W_{x}-v_{x} W_{z}\right)\left(P_{1 y}-P_{2 y}\right)}{2 A_{E} r}\right.} \\
& \left.+\frac{\left(V_{x} W_{y}-V_{y} W_{x}\right)\left(P_{1 z}-P_{2 z}\right)}{2 A_{E} r}\right]
\end{aligned}
$$

The geometrical quantities that appear in the configuration factor relationship for a sma11 triangular element, Eq. (3), are related to the positions in space of the nodal points of the element and the position in space and orientation of the target by Eqs. (4) to (15).

Solution of Eq. (2) is achieved by summing the contribution to the total configuration factor of each of the small triangular elemements that is both contained within the field-of-view of the target and contains the target within its own field-of-view. These elements can be identified by considering the values of $\cos \theta_{1}$ and $\cos \theta_{2}$ depicted on the oblique conical frustrum of elliptical cross section shown in Fig. 2 .

Triangular element $J 1$ is located outside the field-of-view of the target. This is defined by the condition that $\theta_{1}, 90^{\circ}$ or $\cos \theta_{1}<0$. Triangular element $\mathrm{J} 2$ is aligned such that the target cannot be seen from the position and orientation of the element. This is defined by the condition that $\theta_{2}, 90^{\circ}$ or $\cos \theta_{2}: 0$. In both of these cases radiant heat transfer cannot take place between that particular section of the emitting surface and the target in its current position and orientation. 
The solution of $\mathrm{Eq} \cdot(2)$ can, therefore, be written as:

$$
\begin{aligned}
F_{21} & =\sum_{\text {a } 11 \text { elements }} \\
& \text { where } \cos \theta_{1}, 0 \\
& \text { and } \cos \theta_{2}, 0
\end{aligned}
$$

\section{ORIENTATION OF TARGET THAT SUBTENDS THE MAXIMUM CONFIGURATION FACTOR}

of particular interest when studying radiant heat transfer is the orientation of a target at a selected location that is subjected to the maximum incident thermal radiation. When the emission from the surface is assumed to be uniform, this is identical to the orientation of the target that subtends the maximum configuration factor.

Provided the plane surface containing the infinitesimal target does not intersect the emitting surface then the configuration factor for a particular location and orientation of the target can be related to the maximum configuration factor, $\mathrm{F}_{21 \mathrm{MAX}}$, at the same location as follows:

$F_{21}=F_{21 M A X} \cos \phi$

where $\phi$ represents the angle between the normals of the particular target and the target that subtends the maximum configuration factor.

If the plane surface containing the infinitesimal target does intersect the emitting surface then $\mathrm{Eq}$. (17) does not hold, because $\mathrm{F}_{21}$ is evaluated using only that part of the emitting surface contained within the field-ofview of the target. However, if the first condition imposed during the summation in Eq. (16), $\cos \theta_{1}>0$, is relaxed, then a quantity is calculated, $\mathrm{F}_{21}^{\prime}$, whatever orientation of the target is employed, such that:

$$
\begin{aligned}
\mathrm{F}_{21}^{\prime} & =\sum_{\text {a } 11 \text { elements }} \mathrm{F}_{\mathrm{El}} \\
& \text { where } \cos \theta_{2}>0
\end{aligned}
$$

and

$$
F_{21}^{\prime}=E_{21 M A X} \cos \phi
$$

$F_{21}^{\prime}$, un1ike $F_{21}$, can be considered as a vector component of $F_{21 M A X}$ for ali orientations of the target including those in which the plane containing the target intersects the emitting surface.

To determine the maximum configuration factor F 2 IMAX and the orientation of the target that subtends the maximum configuration factor,

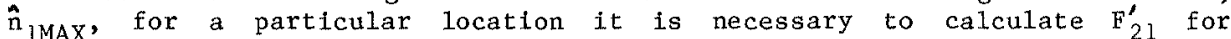
three mutually perpendicular orientations of the target. The most convenient orientations to select are those such that the normals to the target lie parallel to the $X, Y$ and $Z$ axes of the Cartesian co-ordinate system employed. Hence $F_{21 x}^{\prime}, F_{21 y}^{\prime}$ and $F_{21 z}^{\prime}$ are obtained which can be considered as the $x$, ' $Y$ and $Z$ components of $F$ IMAX the magnitude of which is given by: 
$F_{21 M A X}=\left[F_{2 l x}^{\prime 2}+F_{2 l y}^{\prime 2}+F_{2 l z}^{\prime 2}\right]^{\frac{1}{2}}$

and

$\hat{n}_{\text {IMAX }}=\left[\begin{array}{lll}F_{21 x}^{\prime} & \frac{F_{2 l y}^{\prime}}{F_{21 M A X}}, & \frac{F_{2 l z}^{\prime}}{F_{21 M A X}},\end{array}\right]$

\section{APPLICATION TO FLAME SHAPES RELEVANT TO LARGE SCALE FIRES}

The method has been employed to calculate the configuration factor for a solid geometry that can be used to represent a wide range of flame shapes relevant to large scale fires. This geometry is shown in Fig. 2 and in its most general form is an oblique conical frustrum of elliptical cross section. The equation describing the curved surface can be written as:

$\frac{(x-z \tan \alpha)^{2}}{(z \tan \beta+a)^{2}}+\frac{y^{2}}{(z b \tan \beta / a+b)^{2}}=1$

where a represents half the dimension of the base of the flame in the $\mathrm{X}$ direction

b represents half the dimension of the base of the flame in the $\mathrm{X}$ direction

$x, y$ and $z$ represent the $X, Y$ and $Z$ co-ordinates of a point on the curved surface

$\propto$

represents the angle of tilt of the axis from the vertical

B

represents half the cone angle measured in the plane of the $X$ and $Z$ axes and is given by:

$\mathcal{B}=\tan ^{-1} \frac{A-a}{H}$

where A

represents half the dimension of the tip of the flame in the $\mathrm{X}$ direction

H

represents the vertical height of the flame.

By selecting appropriate values for the dimensions contained within Eqs. (22) and (23) various shapes can be generated ranging from a right circular cylinder to an oblique conical frustrum of elliptical cross section as shown in Fig. 3 .

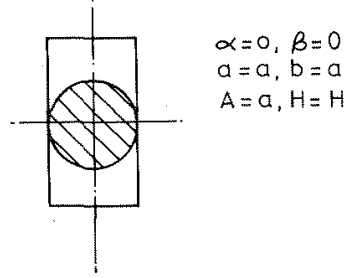

a) Right circular cylinder

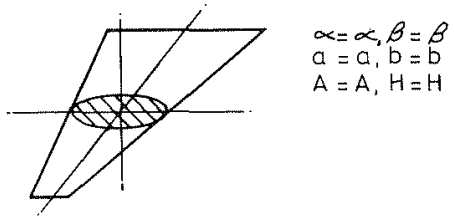

b) Oblique conical frustum of elliptical cross section

FIGURE 3. Wide range of flame shapes relevant to large scale fires. 


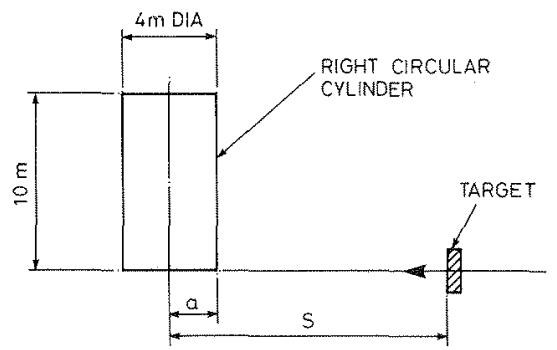

FIGURE 4. Geometrical arrangement used to examine the number of surface elements required to achieve a desired accuracy.

Tilted shapes can also be considered by appropriate positioning and orientation of the receiving target. More complex flame geometries can be formed using combinations from the range of basic shapes shown in Fig. 3 .

Also shown in Fig. 2 is the technique employed to divide the surface into small triangular elements. The number of elements used within the numerical method depends upon the value selected for the integer $\mathrm{N}$.

\section{ACCURACY}

The accuracy of the numerical technique was assessed by comparison with exact analytical solutions for certain simple geometries such as right and oblique circular cylinders ${ }^{8}$ and circular and elliptical plane surfaces 5 . The accuracy of the computations is dependent upon the number of elements into which the surface is divided. Furthermore the number of elements required to achieve a desired accuracy increases as the distance of the target from the emitting surface decreases. This was examined by comparing the results from the numerical technique with the exact analytical solution for a right circular cylinder and a vertical target at ground level and aimed at the centre of the base of the cylinder as shown in Fig. 4.

The number of elements required for a range of different distances, $s$, from the centre line of the cylinder to the target to achieve an accuracy of $\pm 1 \%$ in the evaluation of the configuration factor is shown in Fig. 5 .

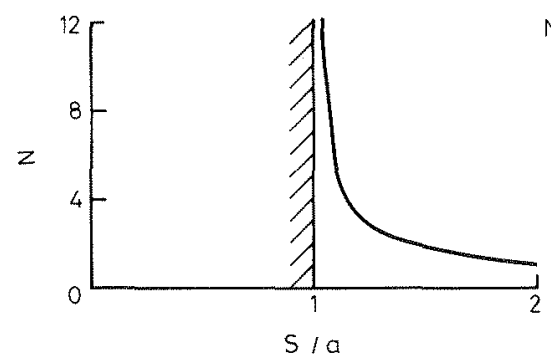

FIGURE 5. Variation of the number of surface elements required to achieve an accuracy of $\pm 1 \%$ in the evaluation of the configuration factor. 


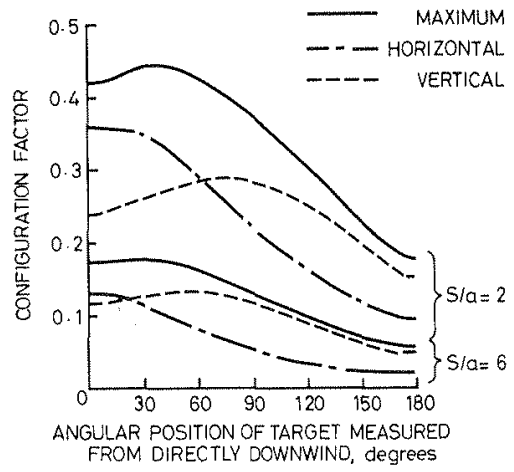

FIGURE 6. Variation of the configuration factor around the oblique conical frustum of elliptical cross section in Fig. 2.

The results shown in FIg. 5 confirm the validity of the computational method. They also show, however, that as the target approaches very close to the emitting surface the number of elements and consequently the computational time required to achleve a certain accuracy rapidly increases.

\section{RESULTS FROM A TYPICAL APPLICATION OF THE METHOD}

The configuration factor has been calculated for a flame shape defined by the oblique conical frustrum of elliptical cross section shown in Fig. 3. Targets were located at ground level at two radial distances from the centre of the elliptical base and at a range of angular positions. Three target orientations were considered. These were vertical such that the normal to the target was horizontal and aimed at the centre of the elliptical base, horizontal such that the normal to the target was vertical and aimed in an upward direction, and finally such that the target was orientated to subtend the maximum configuration factor.

The variation of the configuration factor as the target position changes from directly downwind through $180^{\circ}$ to directly upwind is shown in Fig. 6 .

\section{DISCUSSION}

The numerical technique developed is a general method and is capable of being applied to any solid geometrical shape. This need not necessarily be a regular shape but can comprise of combinations of regular shapes or even completely irregular shapes provided it can be accurately defined. In the case of geometries incorporating concave surfaces additional special conditions will be required to ensure that any particular element is not obscured from the target by other parts of the emitting surface.

The technique has been presented as a method for calculating the configuration factor between an emitting surface and an infinitesimal target. The thermal radiation incident upon a target is then given by Eq. (1) provided the surface emissive power can be considered to be constant and the transmisivity of the atmosphere can be adequately determined from a mean path length for the thermal radiation. However, the method is not restricted by 
these conditions. Large hydrocarbon fires involving fuels such as LPG and kerosene ${ }^{3}$ are very sooty. This obscures large areas of the flame surface which effectively reduces the surface emissive power in those regions and the nett 'result can be a substantial reduction in the incident thermal radiation on objects located outside the flame. Such spatial variations in the flame surface emissive power can easily be incorporated by assigning a particular value to each element. The atmospheric transmisivity for individual elements can also be determined using the distance from the target to the centre-ofarea of each element as the path length for the thermal radiation.

\section{ACKNOWLEDGEMENT}

The Author wishes to thank British Gas Corporation for permission to publish this paper.

\section{REFERENCES}

1. American Gas Association: "LNG Safety Program Phase II - Consequences of LNG Spills on Land," Project 1S-3-1, 1973.

2. Raj, P.K., Moussa, A.N. and Aravamudan, K.: "Experiments Involving Poo1 and Vapour Fires from Spills of Liquified Natural Gas on Water," Report. No. CG-D-55-79, U.S. Department of Transportation, 1979.

3. Mizner, G.A. and Eyre, J.A.: "Large-Scale LNG and LPG Pool Fires," I. Chem. E. Symposium Series No. 71, 1982.

4. Moorhouse, J.: "Scaling Criteria for Pool Fires derived from Large Scale Experiments," I. Chem. E. Symposium Series No. 71, 1982.

5. McGuire, J.H.: Heat Transfer by Radiation, Department of Scientific and Industrial Research and Fire Offices' Comittee, Fire Research Special Report No. 2, London: Her Majesty's Stationary office, 1953.

6. Sparrow, E.M. and Cess, R.D.: Radiation Heat Transfer, Brooks/Cole Publishing Company, 1966.

7. Rein, R.G, Jr., Sliepsevich, C.M. and Welker, J.R.: "Radiation View Factors for Tilted Cylinders," J. Fire and Flammability, Vo1. 1, 1970.

8. Raj, P.K. and Kalelkar, A.S.: "Assessment Models in support of the Hazard Assessment Handbook (CG-446-3)," Technical Report Prepared for the U.S. Coast Guard, NTIS Publication \# AD776617, 1974 . 